\title{
Depression, posttraumatic stress disorder, and grade point average among student servicemembers and veterans
}

\author{
Craig J. Bryan, PsyD, ABPP; ${ }^{*}$ AnnaBelle O. Bryan, BSPH; ${ }^{1-2}$ Kent Hinkson Jr; ${ }^{3}$ Michael Bichrest; ${ }^{4}$ D. Aaron \\ Ahern, PhD $^{5}$ \\ ${ }^{1}$ National Center for Veterans Studies, The University of Utah, Salt Lake City, UT; ${ }^{2}$ Salt Lake Community College, Salt \\ Lake City, UT; ${ }^{3}$ Department of Psychology, Utah Valley University, Provo, UT; ${ }^{4}$ Department of Education, Rivier Uni- \\ versity, Nashua, $\mathrm{NH} ;{ }^{5}$ Veterans Integration To Academic Leadership Program, Cincinnati Department of Veterans \\ Affairs (VA) Medical Center, Cincinnati, OH; and VA Salt Lake City Health Care System, Salt Lake City, UT
}

\begin{abstract}
The current study examined relationships among self-reported depression severity, posttraumatic stress disorder (PTSD) symptom severity, and grade point average (GPA) among student servicemembers and veterans. We asked 422 student servicemembers and veterans (72\% male, $86 \%$ Caucasian, mean age $=36.29 \mathrm{yr}$ ) to complete an anonymous online survey that assessed self-reported GPA, depression severity, PTSD severity, and frequency of academic problems (late assignments, low grades, failed exams, and skipped classes). Female respondents reported a slightly higher GPA than males (3.56 vs 3.41, respectively, $p=0.01$ ). Depression symptoms (beta weight $=-0.174, p=0.03)$, male sex (beta weight $=$ $0.160, p=0.01$ ), and younger age (beta weight $=0.155, p=$ $0.01)$ were associated with lower GPA but not PTSD symptoms (beta weight $=-0.040, p=0.62$ ), although the interaction of depression and PTSD symptoms showed a nonsignificant inverse relationship with GPA (beta weight $=-0.378, p=$ 0.08). More severe depression was associated with turning in assignments late (beta weight $=0.171, p=0.03$ ), failed exams (beta weight $=0.188, p=0.02$ ), and skipped classes (beta weight $=0.254, p=0.01$ ). The relationship of depression with self-reported GPA was mediated by frequency of failed exams. Results suggest that student servicemembers and veterans with greater emotional distress also report worse academic performance.
\end{abstract}

Key words: academics, college, depression, GPA, grade point average, military, posttraumatic stress disorder, student, university, veteran.

\section{INTRODUCTION}

In 2012, just under 1 million U.S. military personnel and veterans received financial assistance from the Department of Veterans Affairs' (VA's) various educational programs, which marks an increase of approximately 75 percent since 2008 [1]. This dramatic increase in the use of educational benefits by military personnel and veterans is due in large part to the Post-9/11 Veterans Educational Assistance Act of 2008, commonly referred to as "the new GI Bill." An additional 121,000 veterans enrolled in college courses through the vocational rehabilitation and employment program, which is intended to

\footnotetext{
Abbreviations: $\mathrm{CI}=$ confidence interval, GPA = grade point average, PCL = Posttraumatic Stress Disorder Checklist, PCL$\mathrm{SF}=$ Posttraumatic Stress Disorder Checklist-Short Form, PHQ-9 = Patient Health Questionnaire-9, PTSD = posttraumatic stress disorder, $\mathrm{SD}=$ standard deviation, VA = Department of Veterans Affairs.

*Address all correspondence to Craig J. Bryan, PsyD, ABPP; National Center for Veterans Studies, The University of Utah, 260 S. Central Campus Dr, Room 205, Salt Lake City, UT 84112; 801-587-7978.

Email: craig.bryan@utah.edu

http://dx.doi.org/10.1682/JRRD.2014.01.0012
} 
assist veterans with certain service-connected disabilities to obtain stable and suitable employment [1]. It is currently unknown how many military personnel and veterans pay for college expenses on their own without accessing VA educational benefits.

Military personnel and veterans who attend college and university courses (i.e., student servicemembers and veterans) may differ from traditional college students in several ways [2-3]. First, student servicemembers and veterans are more likely than traditional college students to be male, older in age, married, and have children [2]. Student servicemembers and veterans also behave differently than traditional students because of the unpredictability of the military system (e.g., deployments, training requirements, reassignments). For instance, student servicemembers and veterans are much more likely to stop attending college completely for a temporary period of time and then resume at a later date [3]. In addition, military personnel and veterans have elevated rates of medical and psychiatric conditions relative to the general population [4]. Up to 75 percent of student servicemembers and veterans have service-connected disabilities, of which the most common is a psychological or emotional condition such as posttraumatic stress disorder (PTSD) or depression [5].

Research among college students suggests that emotional distress is inversely related to academic performance and grades [6]. To date, however, these associations have not been examined among student servicemembers and veterans, a group that may be at elevated risk for anxiety and mood disorders. In a recent sample of student servicemembers and veterans, for instance, 25 percent screened positive for depression and 45 percent screened positive for PTSD [7], rates that are over twice the reported rates among veterans of the conflicts in Iraq and Afghanistan, of whom an estimated 5 to 17 percent meet criteria for PTSD and up to 25 percent report a mental health problem of some kind [8-10]. Research supports a relationship between combat exposure and mental health problems among military personnel [10-11] and student servicemembers and veterans [12]. Given that approximately two-thirds of student servicemembers and veterans report having been in combat while in the military, the relatively high rates of emotional distress among them may be due to military-related stressors. It is also possible that the higher rates of depression and PTSD reported by student servicemembers and veterans relative to military personnel are influenced by contextual demands such as mental health stigma. Specifically, student servicemembers and veterans may be more willing to report emotional distress on surveys conducted external to the military. Although the exact reasons for the higher rates of depression and PTSD among student servicemembers and veterans are not yet clear, it is clear that many student servicemembers and veterans are reporting elevated levels of emotional distress.

Of the anxiety and stressor-related disorders, PTSD has an especially strong relationship with lower academic achievement in general college student populations [13], particularly during the first year of college. Students with PTSD are also more likely to earn a lower grade point average (GPA) during their first year in college, which may partially account for increased attrition among firstyear students who report a history of trauma [14-15]. Depression is also inversely related to academic performance among college students [16-18], perhaps because depressed students experience classroom difficulties such as skipped classes, missed assignments, and failed exams more often than nondepressed students [18]. When depression and anxiety are considered together, depression may have a relatively stronger relationship with academic performance [16], although the combination of depression and anxiety is associated with worse academic performance than each condition alone [19].

Given that PTSD and depression are associated with lower GPA and increased likelihood of early drop out from school, student servicemembers and veterans may be a subgroup of college students at increased risk for experiencing academic difficulties. However, we are unaware of any studies that have examined academic performance among student servicemembers and veterans. In light of this research gap, the primary aim of the current study was to conduct a preliminary examination of the relationships among self-reported PTSD, depression, academic performance, and academic problems in a sample of student servicemembers and veterans who completed an anonymous survey online. We specifically hypothesized that self-reported PTSD and depression symptoms would be associated with lower GPA and more frequent academic problems and that the interaction of PTSD and depression (i.e., greater severity of each) would be associated with significantly lower GPA. 


\section{METHODS}

Participants included 422 college student servicemembers and veterans $(71.9 \%$ male) ranging in age from 19 to $78 \mathrm{yr}$ (mean \pm standard deviation $[\mathrm{SD}]=36.29 \pm$ $10.25 \mathrm{yr}$ ) attending universities and colleges across the United States. Racial distribution was 85.5 percent Caucasian, 5.7 percent African American, 3.3 percent Asian, 3.3 percent Native American, and 1.2 percent Pacific Islander. Hispanic and Latino ethnicity was assessed separately from racial identity and endorsed by 11.6 percent of participants. The majority of participants (64.9\%) were veterans no longer in military service (e.g., retired, honorably discharged); the remaining participants $(35.1 \%)$ reported that they were still serving in one of the military's components. Students represented all branches of service: 32.5 percent Air Force, 37.8 percent Army, 7.7 percent Marine Corps, 20.1 percent Navy, and 1.2 percent Coast Guard. Nearly three-quarters (74.2\%) reported having been deployed at least once while in the military.

\section{Procedures}

Participants were recruited from universities across the United States in coordination with university administrators and personnel working with student servicemembers and veterans during the 2013 calendar year (i.e., spring 2013, summer 2013, and fall 2013 academic terms). An invitation to participate was sent to university veteran services coordinators via a national organizational email listserv. Interested university administrators responded to the researchers with their contact information. Information regarding the study was then emailed to the veteran services coordinators with an embedded hyperlink to the online survey. Coordinators forwarded this message to known student servicemembers and veterans enrolled at their respective universities. Upon visiting the study Web site, student servicemembers and veterans reviewed the informed consent document and, if agreeing to participate, proceeded to the survey. Upon completion of the survey, participants were invited to forward the hyperlink to the study Web site to friends and other student servicemembers and veterans. The survey was completed anonymously and took an average of 12 to $15 \mathrm{~min}$ to complete. A total of 561 individuals accessed the survey Web site, of whom 437 (77.9\%) completed the survey. Of these 437, 15 (3.3\%) indicated that they had never served in the military and were there- fore excluded, leaving 422 student servicemembers and veterans (i.e., $75.2 \%$ of all individuals accessing the survey). Because of the methods employed, it is unknown what proportion of all student servicemembers and veterans who were provided an opportunity to participate (i.e., those who received an email invitation) actually did so. Participants reported being enrolled at the following types of universities and colleges: large (i.e., $>10,000$ students) 4-year public $(n=207,49.0 \%)$, small (i.e., $<$ 3,000 students) to medium (i.e., 3,000-9,999 students) 4year public $(n=20,4.8 \%)$, large 4-year private $(n=16$, $3.9 \%)$, small to medium 4-year private $(n=24,5.8 \%)$, large 2-year public $(n=88,20.8 \%)$, small to medium 2year public $(n=16,3.9 \%)$, and for-profit/online $(n=44$, $10.4 \%$ ); seven participants did not report their university or institution's name.

\section{Measures}

\section{Grade Point Average}

GPA was assessed via self-report using the following item: "What is your current grade point average?" Participants in their first academic term who did not yet have a GPA were directed to leave this item blank. Prior research has indicated that self-reported GPAs have very high correlations with GPAs obtained from school records $(r=0.90 \pm 0.05)[20]$ and that discrepancies between self-reported GPA and school-reported GPA are so small that they do not affect research outcomes [21].

\section{Depression}

Depression symptoms were assessed with the Patient Health Questionnaire-9 (PHQ-9) [22], which is a 9-item self-report measure designed to assess how frequently the respondent has experienced each symptom of major depressive disorder during the past $2 \mathrm{wk}$. The scale is widely used as a reliable and validated measure of depression. Internal consistency for the current sample was 0.91 .

\section{Posttraumatic Stress Disorder}

PTSD symptoms were assessed with the PTSD Checklist-Short Form (PCL-SF) [23], which is an abbreviated 6-item version of the full 17-item PTSD Checklist (PCL) [24]. The PCL-SF's six items were chosen by selecting the two items from each of the Diagnostic and Statistical Manual of Mental Disorders-4th Edition PTSD symptom clusters that correlated highest with the full 
scale score and with its respective symptom cluster total score: intrusive memories and images of the trauma and emotional distress when reminded of the trauma (cluster B), avoidance of activities and situations that remind of the trauma and feeling distant or cut off from others (cluster C), and feeling irritable or angry and feeling super alert (cluster D). The PCL-SF correlates very highly with the full PCL $(r=0.97)$ and is comparable with the full scale in terms of sensitivity and specificity for a diagnosis of PTSD. Internal consistency for the current sample was 0.93 . Participants were asked to identify and consider their most stressful lifetime experience when completing the PCL-SF.

\section{Academic Problems}

To assess academic performance, participants were asked to report the frequency of several classroomrelated problems during the most recent academic term on a scale ranging from 0 (never) to 5 (more than 10 times): turning in an assignment or paper late, receiving a lower grade than expected on (but still passing) an exam or quiz, failing an exam or quiz, and skipping or choosing not to attend class.

\section{RESULTS}

\section{Descriptive Statistics}

Of the 422 participants, $146(34.6 \%)$ were in their first academinc term and therefore did not yet have a GPA to report, leaving $276(65.4 \%)$ available for analysis. Mean \pm SD and intercorrelations of all variables were first calculated (Table 1). Self-reported GPA ranged from 0.20 to 4.00 , with a mean GPA of $3.45 \pm 0.49$. Female students $(3.56 \pm 0.40)$ reported a significantly higher mean GPA than male students $(3.41 \pm 0.52, t(275)=$ $2.610, p=0.01)$, but there was no difference between Caucasian and non-Caucasian students $(t(275)=1.354, p=$ $0.18)$. Current GPA was negatively correlated with both PTSD $(r=-0.13, p=0.04)$ and depression symptom severity $(r=-0.20, p=0.01)$.

Using empirically derived cutoff scores for the PHQ9 and PCL-SF scores, we estimated rates of depression and PTSD among participants. For the PHQ-9, we used a cutoff score of 10 to determine probable depression based on the results of a meta-analysis that found this score best balanced sensitivity and specificity for major depressive disorder [25]. For the PCL-SF, we used a cut- off score of 14 to determine probable PTSD based on the results of Lang and Stein, who identified this score as optimizing sensitivity, specificity, and diagnostic efficiency for PTSD [23]. Using these cutoff scores, 31.7 percent of participants screened positive for depression and 48.4 percent screened positive for PTSD. As displayed in Table 2, 46.0 percent screened positive for neither PTSD nor depression, 22.4 percent screened positive for PTSD only, 5.7 percent screened positive for depression only, and 26.0 percent screened positive for both PTSD and depression.

\section{Associations of Posttraumatic Stress Disorder and Depression Severity with Grade Point Average}

To test the association of PTSD and depression symptom severity with current GPA, generalized linear regression with robust maximum likelihood estimation was used. To aid with interpretation of results, standardized regression coefficients (i.e., beta weights) and structure coefficients were calculated and are reported. Age and sex were entered as covariates due to their significant correlations with GPA. Both PTSD symptom severity $(\beta=$ $-0.151, p=0.01)$ and depression symptom severity $(\beta=$ $-0.209, p<0.001)$ were independently associated with significantly lower GPA, even when controlling for sex and age. When both PTSD and depression symptoms were entered into the regression equation simultaneously, the resulting model was statistically significant $(F$ (4, $254)=5.729, p<0.001)$ and accounted for 8.3 percent of the total variance in GPA (Table 3, model 1). Depression symptoms $(\beta=-0.174, p=0.03)$, male $\operatorname{sex}(\beta=0.160, p=$ $0.01)$, and younger age $(\beta=0.155, p=0.01)$ were significantly associated with lower GPA. In contrast, PTSD symptoms were not associated with GPA $(\beta=-0.040, p=$ 0.62). We next calculated the interaction of PTSD and depression severity and added it to the regression model. The interaction term showed a strong trend toward significance $(\beta=-0.378, p=0.08)$ and explained an additional 1.0 percent of variance in GPA (Table 3 , model 2). The Figure displays the form of this interaction. The Johnson-Neyman test for regions of significance indicated that more severe depression was significantly associated with lower GPA among participants scoring higher than 18 on the PCL-SF.

Mean GPAs with standard errors were next calculated and compared across the four student subgroups. Students with both PTSD and depression had the lowest mean GPA, whereas the three remaining groups had comparable 
Table 1.

Intercorrelations and mean \pm standard deviation $(\mathrm{SD})$ of all variables.

\begin{tabular}{|c|c|c|c|c|c|c|c|c|c|}
\hline Variable & GPA & $\begin{array}{c}\text { Late } \\
\text { Assignment }\end{array}$ & $\begin{array}{c}\text { Low } \\
\text { Grade }\end{array}$ & $\begin{array}{l}\text { Failed } \\
\text { Exam }\end{array}$ & $\begin{array}{l}\text { Skipped } \\
\text { Class }\end{array}$ & Sex & Age & PTSD & Depression \\
\hline$\overline{\text { GPA }}$ & - & & & & & & & & \\
\hline $\begin{array}{l}\text { Late } \\
\text { Assignment }\end{array}$ & $-0.32^{*}$ & - & & & & & & & \\
\hline Low Grade & $-0.33^{*}$ & $0.32^{*}$ & - & & & & & & \\
\hline Failed Exam & $-0.39^{*}$ & $0.36^{*}$ & $0.57^{*}$ & - & & & & & \\
\hline Age & $0.14^{\dagger}$ & $-0.14^{\dagger}$ & $-0.14^{\dagger}$ & -0.03 & $-0.23^{*}$ & -0.07 & - & & \\
\hline PTSD & $-0.13^{\dagger}$ & $0.12^{\dagger}$ & $0.13^{\dagger}$ & $0.15^{\dagger}$ & 0.11 & $0.12^{\dagger}$ & 0.04 & - & \\
\hline Depression & $-0.20^{*}$ & $0.18^{*}$ & $0.18^{*}$ & $0.21^{*}$ & $0.21^{*}$ & 0.08 & 0.01 & $0.68^{*}$ & - \\
\hline$\overline{\text { Mean } \pm \text { SD }}$ & $3.45 \pm 0.49$ & $0.91 \pm 1.29$ & $1.64 \pm 1.38$ & $0.79 \pm 1.28$ & $1.31 \pm 1.49$ & - & $35.45 \pm 9.37$ & $14.83 \pm 7.30$ & $16.66 \pm 6.67$ \\
\hline $\begin{array}{l}{ }^{*} p<0.01 \\
+p<0.05 . \\
\text { GPA }=\text { grade noin }\end{array}$ & PTSD & traumatic & disorder. & & & & & & \\
\hline
\end{tabular}

Table 2.

Student servicemembers and veterans with and without probable posttraumatic stress disorder (PTSD) and depression.

\begin{tabular}{|c|c|c|c|c|}
\hline \multirow{2}{*}{ Variable } & \multicolumn{2}{|c|}{ Depression (-) } & \multicolumn{2}{|c|}{ Depression (+) } \\
\hline & PTSD (-) & PTSD (+) & PTSD (-) & PTSD (+) \\
\hline $\mathrm{GPA}($ mean $\pm \mathrm{SE})$ & $3.50 \pm 0.04^{\mathrm{a}}$ & $3.51 \pm 0.06^{\mathrm{b}}$ & $3.53 \pm 0.10^{\mathrm{c}}$ & $3.27 \pm 0.07^{\mathrm{a}, \mathrm{b}, \mathrm{c}}$ \\
\hline Low Grade $($ mean $\pm \mathrm{SE})$ & $1.48 \pm 0.12^{\mathrm{a}}$ & $1.77 \pm 0.15$ & $1.63 \pm 0.33$ & $1.87 \pm 0.16^{\mathrm{a}}$ \\
\hline Failed Exam (mean $\pm \mathrm{SE})$ & $0.69 \pm 0.11^{\mathrm{a}}$ & $0.77 \pm 0.13$ & $0.47 \pm 0.20^{\mathrm{b}}$ & $1.11 \pm 0.17^{\mathrm{a}, \mathrm{b}}$ \\
\hline Skipped Class (mean $\pm \mathrm{SE}$ ) & $1.14 \pm 0.12^{\mathrm{a}}$ & $1.19 \pm 0.20^{b}$ & $1.48 \pm 0.36$ & $1.80 \pm 0.18^{\mathrm{a}, \mathrm{b}}$ \\
\hline
\end{tabular}

GPAs (Table 2). On average, students with both PTSD and depression reported a GPA that was 0.25 lower than students in all other groups.

\section{Associations of Posttraumatic Stress Disorder and Depression Severity with Academic Problems}

In terms of self-reported academic problems, the majority of participants reported that each problem was experienced relatively infrequently (Table 4). The most commonly occurring problem was receiving a lower than expected grade on a test or exam, which was experienced at least once by 74 percent of participants. Just under half (44\%) of participants turned in an assignment late and 56 percent skipped class at least once. Approximately one-third $(37.6 \%)$ of participants failed an exam at least once during the most recent academic term. As would be expected, current GPA was also negatively correlated with greater frequency of academic problems: turning in assignments late $(r=-0.33, p<0.001)$, earning lower grades than expected $(r=-0.39, p<0.001)$, failing exams $(r=-0.39, p<0.001)$, and skipping class $(r=$ $-0.22, p<0.001)$.

Generalized linear regression was then used to test the association of PTSD and depression severity with self-reported academic problems. Age and sex were again entered as covariates due to their significant correlations with GPA. Table 5 summarizes the results of regression analyses and indicates that more severe depression was associated with significantly greater frequency of three out of the four academic problems assessed: turning in assignments late $(\beta=0.171, p=$ $0.03)$, failing exams $(\beta=0.188, p=0.02)$, and skipping classes $(\beta=0.254, p=0.01)$. Younger age was associated with significantly more frequent late assignments $(\beta=$ $-0.158, p=0.01)$, earning lower grades than expected $(\beta=$ $-0.148, p=0.01)$, and skipping classes $(\beta=-0.253, p<$ 
JRRD, Volume 51, Number 7, 2014

Table 3.

Generalized linear regression coefficients predicting grade point average.

\begin{tabular}{|c|c|c|c|c|c|c|}
\hline Model & $\boldsymbol{R}$ & $R^{2}$ & Predictor & $\boldsymbol{\beta}$ & $p$-Value & $\begin{array}{l}\text { Structure } \\
\text { Coefficient }\end{array}$ \\
\hline \multirow[t]{3}{*}{1} & 0.288 & 0.083 & Sex & 0.160 & 0.01 & 0.476 \\
\hline & & & Age & 0.155 & 0.01 & 0.493 \\
\hline & & & Depression & -0.174 & 0.03 & -0.667 \\
\hline \multirow[t]{3}{*}{2} & 0.305 & 0.093 & Sex & 0.159 & 0.01 & 0.449 \\
\hline & & & Depression & 0.083 & 0.56 & -0.630 \\
\hline & & & PTSD $\times$ Depression & -0.378 & 0.08 & -0.679 \\
\hline \multirow[t]{4}{*}{3} & 0.473 & 0.223 & Sex & 0.097 & 0.10 & 0.296 \\
\hline & & & Age & 0.106 & 0.08 & 0.296 \\
\hline & & & PTSD & -0.017 & 0.82 & 0.272 \\
\hline & & & Skipped Class & 0.004 & 0.95 & -0.474 \\
\hline
\end{tabular}

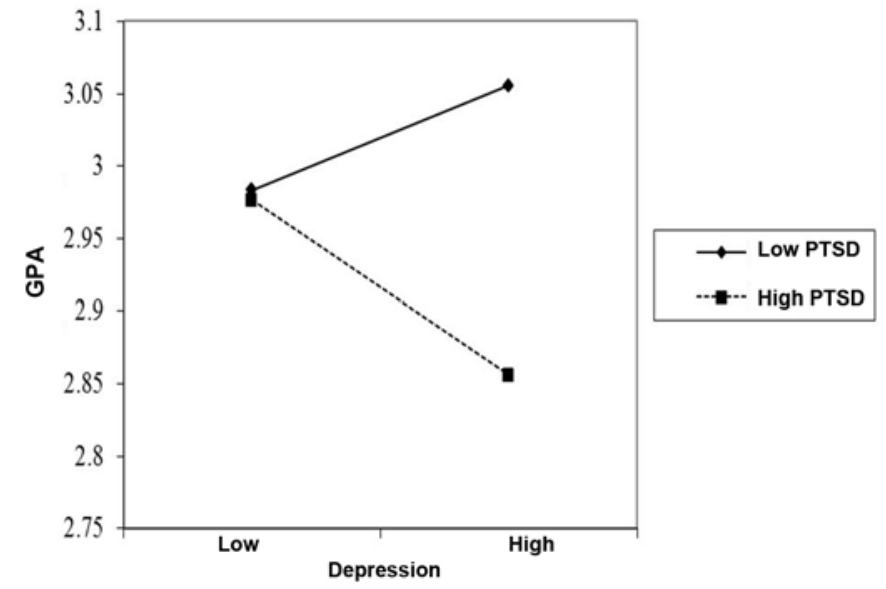

Figure.

Interaction of depression symptom and posttraumatic stress disorder (PTSD) symptom severity on grade point average (GPA) among student servicemembers and veterans, plotted as mean \pm standard deviation scores for depression and PTSD.

$0.001)$. Male students also reported earning lower grades than expected $(\beta=-0.204, p<0.001)$ and failing more exams $(\beta=-0.113, p=0.04)$ than female students. Inspection of the beta weights and structure coefficients indicated that depression severity was among the strongest factors associated with each academic problem. By comparison, the small beta weights and moderately sized structure coefficients for PTSD suggests that although this variable had a moderate association with academic problems, it did not add much predictive value overall, due primarily to its high correlation with depression. With respect to skipping classes, however, younger age showed the relative strongest association. The interaction of PTSD and depression symptom severity was nonsignificant for each of the four academic problems and was therefore excluded from the final models. Comparisons of academic problems across the four subgroups indicated that students with both PTSD and depression reported significantly greater frequency of all four academic problems than students with neither PTSD nor depression (Table 2).

\section{Academic Problems as Mediators of Relationship Between Depression and Grade Point Average}

Given the significant associations of depression with GPA and academic problems identified in the previous analyses, we next sought to explore the possibility that academic problems explained the relationship between depression and GPA. To accomplish this, we entered all four academic problems (i.e., frequency of late assignments, earning lower grades than expected, failing exams, and skipping classes as predictors) to the regression model 
Table 4.

Frequency of self-reported academic problems among college student servicemembers and veterans.

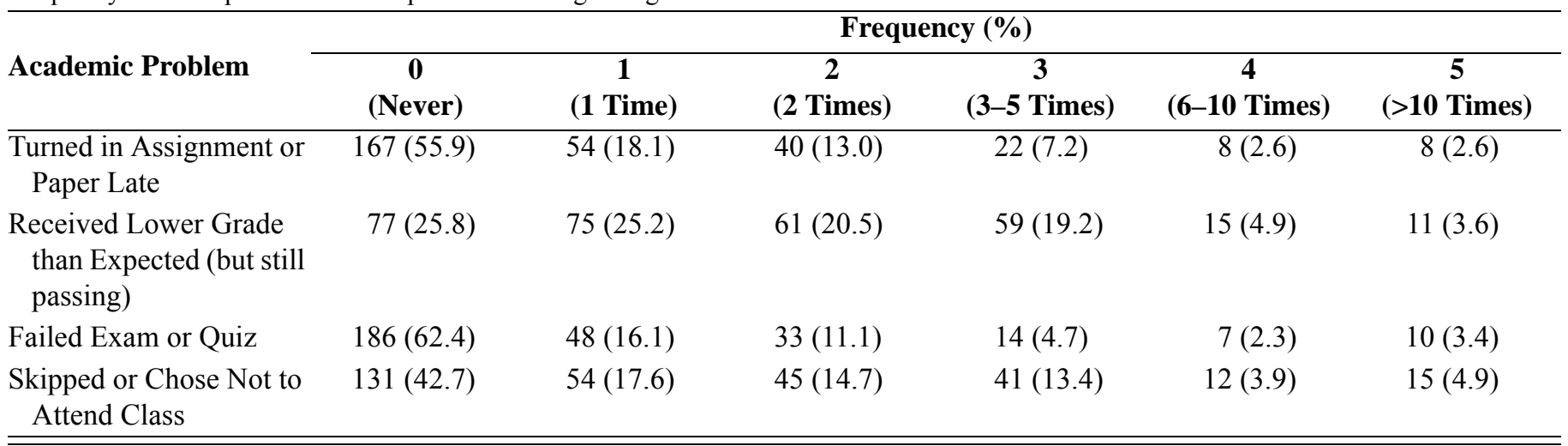

Table 5.

Generalized linear regression coefficients predicting academic problems.

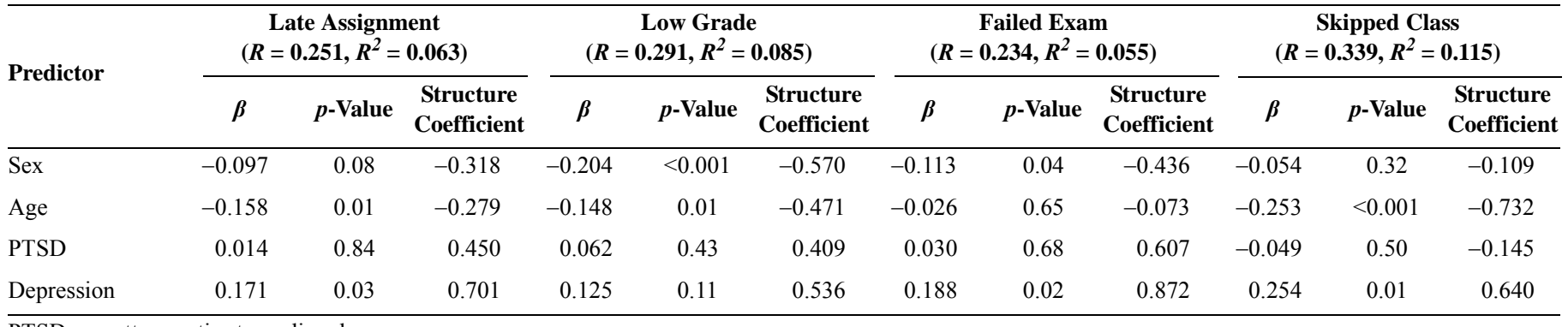

PTSD $=$ posttraumatic stress disorder.

testing the association of PTSD and depression symptom severity with GPA. Table 3 summarizes results (model 3) and suggests that higher GPA was significantly associated with female sex $(\beta=0.097, p=0.10)$, less frequent late assignments $(\beta=-0.178, p=0.01)$, and less frequent failed exams $(\beta=-0.232, p=0.01)$. With the inclusion of these academic problems, depression was no longer significantly associated with overall GPA, although the structure coefficient $(-0.406)$ suggested that depression was nonetheless moderately associated with GPA. To test the mediation hypothesis, we used the bootstrapping procedures described by Preacher and Hayes [26]. A total of 10,000 samples were drawn with bias-corrected and accelerated confidence intervals (CIs). Results suggested that only the indirect effect of failed exams was a significant pathway $(\beta=-0.003$, standard error $=0.002,95 \%$ CI: -0.008 to 0.000 ), suggesting the relationship of depression with GPA occurs primarily because participants with more severe depression tend to fail exams more often.

\section{DISCUSSION}

The current study provides a preliminary overview of academic performance among student servicemembers and veterans, as well as the relationships among academic performance, academic problems, depression, and PTSD. Conclusions based on findings should be made with caution, however, due to the use of self-report methodology, which could be vulnerable to response bias. Arbisi et al., for instance, has recently noted that selfreport measures of depression and PTSD symptoms may result in higher-than-expected false positive rates when used with Iraq and Afghanistan personnel because they also assess more generalized or diffuse emotional distress [27]. Furthermore, when comparing self-reported GPA to actual GPA, individuals tend to inflate rather than underestimate their GPA [20-21,28], with larger magnitude inflation occurring among individuals involved in litigation and legal proceedings, suggesting that adversarial contexts might contribute more to grade inflation than nonadversarial contexts [28]. A more recent meta-analysis 
conducted in academic settings indicates that although 34 percent of college students overestimate their GPA when asked to self-report, with larger inflation occurring among those students with lower actual GPAs, the overall correlation between self-reported and actual GPA among college students was nonetheless very high $(r=0.90$, 90\% CI: 0.82-0.98) [20]. Taken together, these findings suggest that grade inflation may be a limitation to the current study, especially among those student servicemembers and veterans who have the lowest actual GPAs. Results of the current study should therefore be considered preliminary until future studies using alternative methods to self-report, such as structured clinical interviews and scholastic records, can be conducted.

Overall, the mean self-reported GPA of student servicemembers and veterans in the current study suggested good academic performance, with female participants reporting a higher GPA on average than males. Consistent with expectations and prior research among nonmilitary and nonveteran college students [14,16-18], GPA was inversely associated with depression and PTSD symptoms. Results of the current study further suggest depression may play a relatively stronger role than PTSD symptoms. The proportions of student servicemembers and veterans screening positive for probable depression (31.7\%) and PTSD (48.4\%) in the current study were comparable with previously reported rates among college student servicemembers and veterans $(23.7 \%$ screening positive for "severe depression" and $45.6 \%$ screening positive for PTSD) [7] and the general college student population (28.4\% reporting they "felt so depressed it was difficult to function" and $46.4 \%$ reporting "overwhelming anxiety") [29]. Although there is considerable convergence across these studies, these results nonetheless need to be replicated using more rigorous methods (e.g., structured clinical interviews) designed to maximize diagnostic specificity, thereby minimizing false positives that are often characteristic of screening methods.

The interaction of depression and PTSD symptoms observed in the current study, although marginally nonsignificant, aligns with prior research similarly suggesting an augmenting effect of depression and anxiety on college students' academic performance [19] and suggests that student servicemembers and veterans with cooccurring conditions may be especially likely to experience academic challenges. Previous research with student servicemembers and veterans has indicated an interactive effect of depression and PTSD on increased severity of suicidal thoughts and behaviors among student servicemembers and veterans as well [20]. The current results therefore expand upon previous studies by confirming that student servicemembers and veterans with co-occurring depression and PTSD not only have greater clinical needs but are also more likely to experience challenges in daily functioning associated with quality of life (e.g., employment). From a clinical perspective, the current results may provide useful data for engaging student servicemembers and veterans in mental health services. As noted by Bryan and Morrow, conceptualizing mental health treatment from a more functional perspective is more consistent with the values of the military culture and may therefore be more acceptable to military personnel and veterans who generally have negative impressions of the mental healthcare system [30]. For example, clinicians may describe and "market" empirically supported treatments for comorbid PTSD and depression such as prolonged exposure and cognitive processing therapy as methods for improving academic performance [31]. Indeed, results of clinical trials have consistently supported these therapies as highly effective for comorbid PTSD and depression [32], to include military personnel and veterans across eras [32-34].

Our results do not just have implications for clinicians, however. In the current study, depression was also associated with more frequent occurrences of turning in assignments late, earning lower grades than expected, failing exams, and skipping classes. In general, each of these problems was reported relatively infrequently, but student servicemembers and veterans with comorbid depression and PTSD experienced all four problems significantly more often than those with neither depression nor PTSD. The occurrence of any of these problems more than a few times during an academic term may therefore function as potential "warning signs" for emotional distress among student servicemembers and veterans. Faculty members, university staff, and family members may be especially aware of these issues, and therefore could play an important role in detecting at-risk student servicemembers and veterans and encouraging them to seek out appropriate supportive services and/or academic accommodations. Frequency of failing exams may be a particularly important indicator, because it fully mediated the relationship of depression with GPA, suggesting that this particular academic problem may be especially important for understanding the association of depression with academic performance. Taken together, these results 
suggest that student servicemembers and veterans with more severe depression earn a lower overall GPA primarily because they also tend to fail exams more often. Depressed students report turning in assignments late and skipping class more often, but the nonsignificant indirect effects observed in our regression analyses suggest these particular problems may not be as central to the relationship between depression and GPA.

Results of the current study must be considered within the context of its limitations, which extend beyond the self-report methodology already discussed. For instance, our study's sample is not necessarily representative of all student servicemembers and veterans enrolled in college or university courses. Furthermore, a selfselection bias may exist due to our recruitment strategy, which could limit the generalizability of our conclusions. The current results should therefore be considered preliminary until additional research can replicate these findings. Future efforts should be characterized by the following: (1) use of structured diagnostic interviews in addition to self-report surveys to provide more reliable assessments of depression and PTSD, (2) use of GPA and other indicators of academic performance based on scholastic records rather than self-report, and (3) sampling methods that are more representative of the population and do not rely solely on self-selection. Finally, our cross-sectional design limits our ability to identify the direction of effect among emotional distress and academic performance in student servicemembers and veterans. Specifically, we are unable to determine whether emotional distress serves as a precursor to declines in academic performance or whether poor academic performance precedes emotional distress. Future studies that incorporate longitudinal designs are needed to better understand the temporal relationships among psychological and academic variables in this population.

\section{CONCLUSIONS}

Despite these limitations, the current study provides important information about the academic performance of student servicemembers and veterans and is the first to examine the relationships among depression, PTSD, and academic performance in this population, with overall results suggesting that depression and PTSD may have negative implications for academic success in this group.

\section{ACKNOWLEDGMENTS}

\section{Author Contributions:}

Study concept and design: C. J. Bryan, A. O. Bryan, D. A. Ahern. Acquisition of data: C. J. Bryan, A. O. Bryan, K. Hinkson Jr, M. Bichrest, D. A. Ahern.

Analysis and interpretation of data: C. J. Bryan.

Drafting of manuscript: C. J. Bryan, A. O. Bryan, K. Hinkson Jr, M. Bichrest, D. A. Ahern.

Financial Disclosures: The authors have declared that no competing interests exist.

Funding/Support: This material was unfunded at the time of manuscript preparation.

Institutional Review: Approval for the current study was provided by the University of Utah Institutional Review Board.

Participant Follow-Up: The authors have no plans to notify the study subjects of the publication of this article because of a lack of contact information.

\section{REFERENCES}

1. Department of Veterans Affairs. Annual benefits report. Fiscal year 2012. Washington (DC): Department of Veterans Affairs; 2012.

2. VA Campus Toolkit Handout [Internet]. Characteristics of student veterans. Washington (DC): Department of Veterans Affairs; 2012 [updated 2012 Apr 6; cited 2014 Mar 25]. Available from: http://www.mentalhealth.va.gov/studentveteran/ docs/ed todaysStudentVets.html

3. Education Working Group. Educational attainment: Tracking the academic success of servicemembers and veterans. New York (NY): Servicemembers Opportunity Colleges; 2012.

4. Kessler RC, Heeringa SG, Stein MB, Colpe LJ, Fullerton CS, Hwang I, Naifeh JA, Nock MK, Petukhova M, Sampson NA, Schoenbaum M, Zaslavsky AM, Ursano RJ; Army STARRS Collaborators. Thirty-day prevalence of DSM-IV mental disorders among nondeployed soldiers in the US Army: Results from the Army Study to Assess Risk and Resilience in Servicemembers (Army STARRS). JAMA Psychiatry. 2014;71(5):504-13. [PMID:24590120] http://dx.doi.org/10.1001/jamapsychiatry.2014.28

5. Vance ML, Miller WK. Serving wounded warriors: Current practices in postsecondary education. J Postsecondary Educ Disabil. 2009;22:18-35.

6. De Meuse KP. The relationship between life events and indices of classroom performance. Teach Psychol. 1985; 12:146-49. http://dx.doi.org/10.1207/s15328023top1203 8

7. Rudd MD, Goulding J, Bryan CJ. Student veterans: A national survey exploring psychological symptoms and suicide risk. Prof Psychol Res Pr. 2011;42:354-60.

http://dx.doi.org/10.1037/a0025164 
8. Hoge CW, Castro CA, Messer SC, McGurk D, Cotting DI, Koffman RL. Combat duty in Iraq and Afghanistan, mental health problems, and barriers to care. N Engl J Med. 2004; 351(1):13-22. [PMID:15229303] http://dx.doi.org/10.1056/NEJMoa040603

9. Milliken CS, Auchterlonie JL, Hoge CW. Longitudinal assessment of mental health problems among active and reserve component soldiers returning from the Iraq war. JAMA. 2007;298(18):2141-48. [PMID:18000197] http://dx.doi.org/10.1001/jama.298.18.2141

10. Tanielian T, Jaycox L. Invisible wounds of war: Psychological and cognitive injuries, their consequences, and services to assist recovery. Santa Monica (CA): RAND Corporation; 2008.

11. Castro CA, McGurk D. The intensity of combat and behavioral health status. Traumatology. 2007;13:6-23. http://dx.doi.org/10.1177/1534765607309950

12. Rudd MD. Severity of combat exposure, psychological symptoms, social support, and suicide risk in OEF/OIF veterans. J Consult Clin Psychol. Forthcoming.

13. Kessler RC, Foster CL, Saunders WB, Stang PE. Social consequences of psychiatric disorders, I: Educational attainment. Am J Psychiatry. 1995;152(7):1026-32. [PMID:7793438]

14. Bachrach RL, Read JP. The role of posttraumatic stress and problem alcohol involvement in university academic performance. J Clin Psychol. 2012;68(7):843-59. [PMID:22589016] http://dx.doi.org/10.1002/jclp.21874

15. Duncan RD. Childhood maltreatment and college drop-out rates: Implications for child abuse researchers. J Interpers Violence. 2000;15:987-95. http://dx.doi.org/10.1177/088626000015009005

16. Andrews B, Wilding JM. The relation of depression and anxiety to life-stress and achievement in students. Br J Psychol. 2004;95(4):509-21. [PMID:15527535] http://dx.doi.org/10.1348/0007126042369802

17. Haines ME, Norris MP, Kashy DA. The effects of depressed mood on academic performance in college students. J Coll Student Dev. 1996;37:519-26.

18. Hysenbegasi A, Hass SL, Rowland CR. The impact of depression on the academic productivity of university students. J Ment Health Policy Econ. 2005;8(3):145-51. [PMID:16278502]

19. Eisenberg D, Golberstein E, Hunt JB. Mental health and academic success in college. B E J Econom Anal Policy. 2009;9(1). http://dx.doi.org/10.2202/1935-1682.2191

20. Kuncel NR, Crede M, Thomas LL. The validity of selfreported grade point averages, class ranks, and test scores: A meta-analysis and review of the literature. Rev Educ Res. 2005;75:63-82. http://dx.doi.org/10.3102/00346543075001063
21. Cole J, Rocconi L, Gonyea R. Accuracy of self-reported grades: Implications for research. Proceedings of the 51st Annual Forum Association for Institutional Research; 2012 Jun 5; New Orleans, LA.

22. Kroenke K, Spitzer RL, Williams JB. The PHQ-9: Validity of a brief depression severity measure. J Gen Intern Med. 2001;16(9):606-13. [PMID:11556941] http://dx.doi.org/10.1046/j.1525-1497.2001.016009606.x

23. Lang AJ, Stein MB. An abbreviated PTSD checklist for use as a screening instrument in primary care. Behav Res Ther. 2005;43(5):585-94. [PMID:15865914] http://dx.doi.org/10.1016/j.brat.2004.04.005

24. Weathers F, Litz B, Herman D, Huska J, Keane T. The PTSD Checklist (PCL): Reliability, validity, and diagnostic utility. Proceedings of the Annual Convention of the International Society for Traumatic Stress Studies; 1993 Nov; San Antonio, TX.

25. Manea L, Gilbody S, McMillan D. Optimal cut-off score for diagnosing depression with the Patient Health Questionnaire (PHQ-9): A meta-analysis. CMAJ. 2012;184(3): E191-96. [PMID:22184363] http://dx.doi.org/10.1503/cmaj.110829

26. Preacher KJ, Hayes AF. Asymptotic and resampling strategies for assessing and comparing indirect effects in multiple mediator models. Behav Res Methods. 2008;40(3): 879-91. [PMID:18697684] http://dx.doi.org/10.3758/BRM.40.3.879

27. Arbisi PA, Kaler ME, Kehle-Forbes SM, Erbes CR, Polusny MA, Thuras P. The predictive validity of the PTSD Checklist in a nonclinical sample of combat-exposed National Guard troops. Psychol Assess. 2012;24(4):1034-40. [PMID:22545697] http://dx.doi.org/10.1037/a0028014

28. Greiffenstein MF, Baker WJ, Johnson-Greene D. Actual versus self-reported scholastic achievement of litigating postconcussion and severe closed head injury claimants. Psychol Assess. 2002;14(2):202-8. [PMID:12056082] http://dx.doi.org/10.1037/1040-3590.14.2.202

29. American College Health Association. 2010 national college health assessment. Washington (DC): American College Health Association; 2011.

30. Bryan CJ, Morrow CE. Circumventing mental health stigma by embracing the warrior culture: Lessons learned from the Defender's Edge Program. Prof Psychol Res Pr. 2011;42:16-23. http://dx.doi.org/10.1037/a0022290

31. Institute of Medicine; Committee on the Assessment of Ongoing Efforts in the Treatment of Posttraumatic Stress Disorder. Treatment for posttraumatic stress disorder in military and veteran populations: Initial assessment. Washington (DC): The National Academies Press; 2012.

32. Resick PA, Schuster-Wachen J, Mintz J, Wilkinson C, Dondanville K, Pruiksma K, Borah A, Peterson AL; STRONG 
STAR Consortium. CPT vs. PCT: Final results of a randomized controlled trial. Proceedings of the Annual Meeting of the International Society for Traumatic Stress Studies; 2013 Nov; Philadelphia, PA.

33. Thorp SR, Stein MB, Jeste DV, Patterson TL, Wetherell JL. Prolonged exposure therapy for older veterans with posttraumatic stress disorder: A pilot study. Am J Geriatr Psychiatry. 2012;20(3):276-80. [PMID:22273763] http://dx.doi.org/10.1097/JGP.0b013e3182435ee9

34. Tuerk PW, Yoder M, Grubaugh A, Myrick H, Hamner M, Acierno R. Prolonged exposure therapy for combat-related posttraumatic stress disorder: An examination of treatment effectiveness for veterans of the wars in Afghanistan and Iraq. J Anxiety Disord. 2011;25(3):397-403. [PMID:21131170]

http://dx.doi.org/10.1016/j.janxdis.2010.11.002
Submitted for publication January 19, 2014. Accepted in revised form April 29, 2014.

This article and any supplementary material should be cited as follows:

Bryan CJ, Bryan AO, Hinkson K Jr, Bichrest M, Ahern DA. Depression, posttraumatic stress disorder, and grade point average among student servicemembers and veterans. J Rehabil Res Dev. 2014;51(7):1035-46. http://dx.doi.org/10.1682/JRRD.2014.01.0012

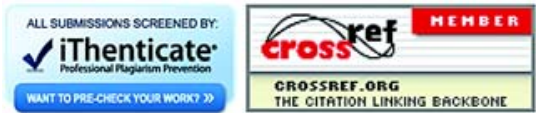


